\title{
Tucson Critical Care Journal Club: Albumin Use in the Critical Care Unit
}

\section{Lyu PF, Hockenberry JM, Gaydos LM, Howard DH, Buchman TG, Murphy DJ. Impact of a sequential intervention on albumin utilization in critical care. Crit Care Med. 2016 Jul;44(7):1307-13. [CrossRef] [PubMed]}

Albumin use for volume resuscitation in critically ill patients has been a hotly contested topic for decades (1). Widespread use of this expensive medicine in some settings has been based on perceived benefits without strong supporting evidence (2). Providing patient centered, evidence based, high value care is an expectation of most healthcare entities thus leading many hospitals to consider the cost effectiveness of their practices (3). One area of interest involves the use of albumin for volume resuscitation. Provider education has not changed practice; therefore, novel approaches to align provider behavior with evidence-based standards are necessary (4-6).

In this pre-post quasi-experimental study with historical controls, investigators at Emory Critical Care Center (ECCC) hypnotized that a sequentially implemented multifaceted intervention would reduce albumin use without increasing mortality. The study was conducted between September 2011 and August 2014 in eight intensive care units (ICUs; two medical, two cardiothoracic, two neuroscience, one surgical, and one coronary unit). The study population included all ICU admissions (ICU readmissions and liver transplant patients excluded).

The first year was an observation period to generate pre-intervention data. In year one of the intervention period, a monthly albumin utilization feedback report was distributed to all units while the intensivists, nurse practitioners and physician assistants received financial incentives to reduce albumin use. Both the financial incentive and the feedback reports ended after the first intervention year.

In the second intervention year, evidence based guidelines for albumin use were incorporated in the computerized ordering system such that providers had to select an indication for albumin use.

A total of 22,004 admissions were included, 7,303 in the baseline year, and 14,701 the 2-year intervention period. Patient characteristics were similar between the two periods. After adjusting for differences in patient characteristics between the baseline and intervention periods, the intervention was associated with a $42 \%$ decrease in the number of albumin orders per ICU admission $(p<0.001)$. This adjusted effect included a relative $18 \%$ reduction in the probability of the patient receiving any albumin order $(p<$ 0.001 ) and a relative $29 \%$ reduction in the number of orders per admission among patients receiving at least one order $(p<0.001)$. The team estimated that reductions in albumin orders translated into mean savings of $\$ 171$ per admission while aggregate direct cost savings were nearly $\$ 2.5 \mathrm{M}$ in the 2-year period. Adjusted analysis found no difference in ICU mortality $(p=0.96)$ or in-hospital mortality $(p=0.96)$. The study concluded that the intervention reduced albumin use without compromising patient safety. 
This study was done in five different ICU settings, which provided great generalizability, however, this can mask variable effects in different units. Even though the preintervention and post-intervention groups had comparable characteristics, the pre-post design provides weaker evidence than randomized controlled trials (RCTs) due to the lack of randomization, which can threaten the internal validity. During the 3-year intervention period, external factors such as new evidence or changes in guidelines in the medical literature, concurrent quality projects or new hospital policies could impact the trends of albumin use.

Given that the study did not collect data on the substitute fluids and related medications, money spent on incentives, providing a comprehensive cost-effectiveness analysis is not possible. It was interesting to see how the monetary incentives reduced average albumin use but did not change who received it, while the guidelines and integration in the ordering process appeared to impact both.

In summary, in this study, the investigators provided a unique perspective on how sequential multi faceted initiative can lead to practice change towards high value care and significant healthcare dollar savings without adversely affecting ICU or hospital mortality. It remains to be seen if this model can be replicated in other settings and if the newly developed habits are sustainable.

Huthayfa Ateeli MBBS, Christian Bime MD and Joe Gerald PhD University of Arizona

Tucson, AZ

\section{References}

1. Patel A, Laffan MA, Waheed U, Brett SJ. Randomised trials of human albumin for adults with sepsis: systematic review and meta-analysis with trial sequential analysis of all-cause mortality. BMJ. 2014 Jul 22;349:g4561. [CrossRef] [PubMed]

2. American Thoracic Society. Evidence-based colloid use in the critically ill: American Thoracic Society Consensus Statement. Am J Respir Crit Care Med. 2004;170(11):1247-1259. [CrossRef] [PubMed]

3. Institute for Healthcare Improvement. IHI triple aim initiative. Available at: http://www.ihi.org/Engage/Initiatives/TripleAim/Pages/Participants.aspx (accessed 11/11/16).

4. Berenholtz S, Pronovost PJ. Barriers to translating evidence into practice. Curr Opin Crit Care. 2003 Aug;9(4):321-5. [CrossRef] [PubMed]

5. Hewson-Conroy KM, Burrell AR, Elliott D, Webb SA, Seppelt IM, Taylor C, Glass P. Compliance with processes of care in intensive care units in Australia and New Zealand--a point prevalence study. Anaesth Intensive Care. 2011 Sep;39(5):926-35. [PubMed]

6. Grol R, Grimshaw J. From best evidence to best practice: effective implementation of change in patients' care. Lancet. 2003 Oct 11;362(9391):1225-30. [CrossRef] [PubMed] 